\title{
Formulación de un nuevo concepto de confiabilidad operacional
}

\author{
Formulating a new concept of operational reliability
}

\author{
Armando Díaz Concepción ${ }^{1 *}$ \\ Alfredo del Castillo Serpa ${ }^{2} \quad$ Jesús Cabrera Gómez ${ }^{1}$ \\ Reynaldo Benítez Montalvo ${ }^{3}$ \\ Lesis Villar Ledo ${ }^{4}$ \\ Alberto Julio Rodríguez Piñeiro ${ }^{1}$
}

Recibido 14 de marzo de 2019, aceptado 12 de enero de 2020

Received: March 14, 2019 Accepted: January 12, 2020

\begin{abstract}
RESUMEN
En la actualidad el mantenimiento ha pasado de una concepción reactiva a una proactiva en donde la confiabilidad operacional es una de las nuevas estrategias que se implementa. En este trabajo se resume la investigación realizada para construir un nuevo concepto de confiabilidad operacional. Se utilizaron los métodos de análisis bibliográfico y encuesta. Para la validación del nuevo concepto formulado fueron consultados destacados especialistas seleccionados con perfiles en Microsoft Academic Search, Google Académico y ResearchGate. Fue diseñado un instrumento tipo encuesta empleando el método Likert y para el análisis de la confiabilidad se utilizó el Alpha de Cronbach. Como resultado, se definió y validó un nuevo concepto de confiabilidad operacional que propugna la acción sinérgica entre el equipamiento, el recurso humano y el proceso tecnológico. Este concepto proporciona las bases para la obtención de un indicador que represente numéricamente a la confiabilidad operacional.
\end{abstract}

Palabras clave: Confiabilidad operacional, concepto, encuesta, validación, equipamiento, recurso humano, proceso.

\begin{abstract}
Maintenance approaches have been evolving from reactive to a preferred proactive conception with operational reliability as a new implemented strategy. This scenario suggests that a review of conceptual basis is needed. The paper resumes the study carried out with the main objective of building a new concept of operational reliability. Bibliographic research and poll methods were used. Selected specialists with remarkable profiles in Microsoft Academic Search, Google Schoolar and Research Gate were consulted in order to validate the formulated concept. A poll instrument based on Likert Method was designed whereas for analyzing the confidence, Cronbach Alpha Method was used. As a result, a novel concept of operational reliability was built and validated. This concept sets up a synergic based relationship among equipment, human resources and technological processes and, for the first time, provides support to obtain an indicator as a numerical representation of operational reliability.
\end{abstract}

Keywords: Operational reliability, concept, poll instrument, validation, equipment, human resource, process ability, concept, poll instrument, validation, equipment, human resource, process.

1 Universidad Tecnológica de La Habana. Centro de Estudios de Ingeniería de Mantenimiento, CEIM. La Habana, Cuba.

E-mail: adiaz@ceim.cujae.edu.cu; jcabrera@ceim.cujae.edu.cu; alberto@mecanica.cujae.edu.cu

2 Universidad Tecnológica de La Habana. Centro de Estudios de Matemática, CEMAT. La Habana, Cuba.

E-mail: acastillo@cemat.cujae.edu.cu

3 Centro de Inmunología Molecular, CIM. La Habana, Cuba. E-mail: reynaldo@ cim.sld.cu

4 Universidad Tecnológica de La Habana. Facultad de Ingeniería Industrial. La Habana, Cuba. E-mail: leisis@ ind.cujae.edu.cu

* Autor de correspondencia: adiaz@ceim.cujae.edu.cu 


\section{INTRODUCCIÓN}

En la actualidad la actividad de mantenimiento ha pasado de una concepción reactiva a una proactiva que tiene al negocio y al cumplimento de la demanda del cliente en su enfoque fundamental, observándose que una de las estrategias que se imponen es la confiabilidad operacional [1].

Diferentes autores definen la confiabilidad operacional como la capacidad de un sistema formado por equipos, procesos, tecnologías y personas para cumplir las funciones para las cuales ha sido concebido, dentro de ciertos límites y para un contexto operacional dado [2].

En la investigación corporativa realizada por Mitos del Mantenimiento, Pensamiento \& Errores, Parte II: Mejorando la Confiabilidad Operacional \& Disponibilidad [3], se discuten aspectos que afectan la obtención de resultados positivos en el mantenimiento de equipos, presentando la confiabilidad operacional como una forma de transformación de la organización, la cual requiere de tiempo y recursos para alcanzar un cambio efectivo en los indicadores de eficiencia de la empresa. Sin embargo, en este trabajo no se propone un concepto de la confiabilidad operacional.

En la metodología propuesta por los autores Stenström, Parida y otros [4], para estimar la disponibilidad y la mantenibilidad como mejora de la confiabilidad operacional, proponen modelos para evaluar la disponibilidad y la mantenibilidad de la organización en forma independiente, aunque se brinda un criterio de integración de ambos para apoyar la toma de decisiones. Proponen que esta metodología solo debe ser usada en sistemas complejos y en entidades con alta tecnología, teniendo como limitación que no analizan los restantes elementos integrantes, además de no pronunciarse por un concepto de confiabilidad operacional.

Los autores Zhang Ding y Zhang Yingjie [5], presentan un análisis de confiabilidad y mantenimiento de los sistemas de fabricación en talleres de trabajo. Partiendo de este análisis, proponen una política eficiente de mantenimiento preventivo en términos de análisis de efectos de falla. Brindan diferentes modelos típicos para análisis cuantitativos de la confiabilidad de equipos y la mantenibilidad, pero no se muestra un indicador que integre a ambas y tampoco proponen un concepto de confiabilidad operacional.

De la investigación realizada se puede concluir que los autores analizados proponen mejoras a la confiabilidad operacional sin formular un concepto que la defina.

El objetivo de la investigación se basó en construir un concepto de confiabilidad operacional. Este concepto pretende integrar en forma efectiva los elementos componentes de la confiabilidad operacional: equipamiento, el recurso humano y el proceso tecnológico. Se propuso una matriz que muestra cómo ha de ser esta integración. Para cumplir con este objetivo se empleó el método de la tormenta de ideas y se utilizaron encuestas para la validación del concepto propuesto. A partir del juicio de los especialistas consultados y los resultados de las encuestas, se llegó a la conclusión de que el concepto que se propuso es adecuado y su contenido supera a los restantes conocidos.

\section{MÉTODOS}

Para la realización de este trabajo se utilizaron los siguientes métodos: análisis bibliográfico y encuesta.

\section{Análisis bibliográfico}

De las publicaciones analizadas se seleccionaron las que se describen a continuación:

- Existen diferentes autores que han escrito y presentado conceptos sobre confiabilidad operacional como: PEMEX Exploración y Perforación [6] que la define como: "un modelo compuesto por 4 elementos, que en su integración va a mantener niveles competitivos en costos de descubrimiento y desarrollo, así como de producción". Esta definición se fundamenta en el análisis de 7 elementos base y 23 subelementos pero no expresa cómo debe ser esta integración.

- Huerta [7] la define como: "capacidad de un sistema formado por equipos, procesos, tecnologías y personas para cumplir las funciones para las cuales ha sido concebido, dentro de ciertos límites y para un contexto operacional dado". Esta definición expone la necesidad de que exista integración entre los elementos integrantes de la confiabilidad, pero no expresa como debe ser la misma y tampoco como cuantificarla. 
- Parra [8] la define como: "capacidad de una instalación o sistema (integrado por procesos, tecnología y gente), para cumplir su función dentro de sus límites de diseño y bajo un contexto operacional específico". En esta definición se expresa la necesidad de integrar los diferentes elementos que componen a la confiabilidad operacional así como la importancia de tener presente las funciones y el contexto, pero no indica cómo debe realizarse esta integración así como tampoco expresa la posibilidad de su cuantificación.

- García Palencia, en varias de sus obras [2, 9] la define como "una serie de procesos de mejora continua que incorporan, en forma sistemática, avanzadas herramientas de diagnóstico, metodologías de análisis y nuevas tecnologías, en búsqueda de optimizar la gestión, planeación y control de la producción industrial". Plantea que para su aplicación efectiva, es imprescindible lograr que los 5 elementos integrantes (confiabilidad de los activos, del diseño, de los procesos, humana y clase mundial), sean parte integral de la organización y no limitarse a la actividad de mantenimiento. En el concepto planteado se demuestra la necesidad de la integración de los elementos componentes, pero no se expresa como debe ser esta integración. También plantea que en el análisis de mejora de la confiabilidad operacional se pudiera optimizar la gestión de la producción industrial, pero sin la posibilidad de una cuantificación se hace muy difícil realizar este proceso al no disponer de un indicador que involucre a los elementos componentes de la confiabilidad operacional.

- Se pudo apreciar que cada autor en las obras antes citadas presenta una matriz conformada por los elementos componentes de la confiabilidad operacional que respalda cada concepto.

- A partir del análisis de los 4 conceptos antes expuestos, se puede plantear que los autores concuerdan en que un proceso de mejora de la confiabilidad operacional debe sustentarse sobre la base de analizar diferentes elementos tales como: el equipamiento, los procesos, el mantenimiento y los recursos humanos. Estos elementos deben estar integrados para el análisis de la confiabilidad operacional.

- Además, proponen la utilización de indicadores para la evaluación de los elementos integrantes a través de diferentes modelos matemáticos, pero la evaluación de la confiabilidad operacional la realizan bajo criterios cualitativos. En todos los casos, la limitación de mayor impacto es que no expresan la posibilidad de cuantificar la confiabilidad operacional, integrando los elementos que la componen [12].

\section{Instrumento para validar el concepto}

Para la validación del concepto de confiabilidad operacional se diseñó un instrumento tipo encuesta $[12,13]$ y para la confección de la misma se utilizó la consulta de expertos.

La encuesta quedó constituida por los siguientes elementos:

- El tipo de investigación fue transeccional o transversal, ya que recogió datos en un solo momento y en un tiempo único, partiendo de la descripción de las variables y analizando su incidencia en un momento dado [12].

- El tipo de muestra seleccionada fue la de sujetos-tipo debido a que es la apropiada para estudios exploratorios o en investigaciones del tipo cualitativo [11, 12]. Para la investigación se tomó una muestra a partir de una población que incluyó:

- Especialistas internacionales y nacionales de alto nivel en el tema de la confiabilidad operacional, con alta cantidad de citas e índice h seleccionados a partir de sus perfiles en Google Académico y ResearchGate. Todos con artículos publicados en la WoS, Scopus y Scielo.

- Otros especialistas nacionales e internacionales reconocidos en el tema de la confiabilidad operacional por su desempeño en la actividad como consultores de diferentes importantes empresas y universidades.

En total se seleccionaron 37 especialistas, tanto nacionales como internacionales, a los que se les envió la encuesta de validación del concepto, se recibió respuesta de 22 de estos especialistas para un 59,5\%. La composición de los mismos es la siguiente:

- Nacionalidad

- Especialistas nacionales: 9

- Especialistas extranjeros: 13

- Grado científico

- Doctores en Ciencias: 7

- Másteres en Ciencias: 15 
Para el diseño de la encuesta se utilizó el Método Likert (14) y para la medición de la confiabilidad de la misma se empleó el Método de Estimación Alfa de Cronbach [14-15], calculando este valor de confiabilidad para todo el instrumento. Para el análisis de los resultados obtenidos de la variable de salida, los indicadores y las dimensiones se utilizó el promedio de los valores dados por los expertos. El procesamiento de la información recogida se realizó con la ayuda del software MiniTab. Además, se agregó en la encuesta una pregunta abierta para que los especialistas expusieran cualquier criterio que consideraran necesario.

El diseño del instrumento quedó conformado por:

- 1 variable de salida: validez del concepto de confiabilidad operacional.

- 2 dimensiones: pertinencia y precisión.

- 4 indicadores: contextualización, actualidad, claridad y concordancia.

Como se describe en la Tabla 1.

\section{RESULTADOS Y DISCUSIÓN}

\section{Definición del concepto de confiabilidad operacional}

En la definición del concepto de confiabilidad operacional formulado y evaluado se hace referencia al Sistema Técnico Complejo. Este sistema está integrado por diferentes componentes autónomos, pero interrelacionados, interdependientes y vinculados entre sí. Presenta propiedades que surgen de la interacción entre sus partes, las que no pueden predecirse a partir de las propiedades de las partes [16].

Entonces, basado en la investigación bibliográfica realizada en este trabajo y en [6], la consulta a los expertos y la experiencia de los autores, el concepto de confiabilidad operacional queda definido como sigue:
Concepto. La confiabilidad operacional es la acción sinérgica del equipamiento, el recurso humano y el proceso tecnológico, para lograr que un sistema técnico complejo cumpla las funciones requeridas en un tiempo y contexto operacional determinados. Puede ser expresada a través de un indicador.

En la Figura 1 se muestran los elementos de la matriz que sustenta este concepto de confiablidad operacional.

Esta nueva definición expresa, en forma explícita, que no es cualquier tipo de integración la que debe existir entre los elementos constitutivos de la confiabilidad operacional. Cuando expresa que es la acción sinérgica de los 3 elementos a los que se refiere, deja claramente establecido que estos elementos necesariamente han de tener un aporte a la confiabilidad operacional, a diferencia de lo expresado en la bibliografía consultada $[6,7,8,9$ y 10]. También deja claro que puede ser representada a través de un valor numérico y los análisis que se deriven de este pueden apoyar a las organizaciones

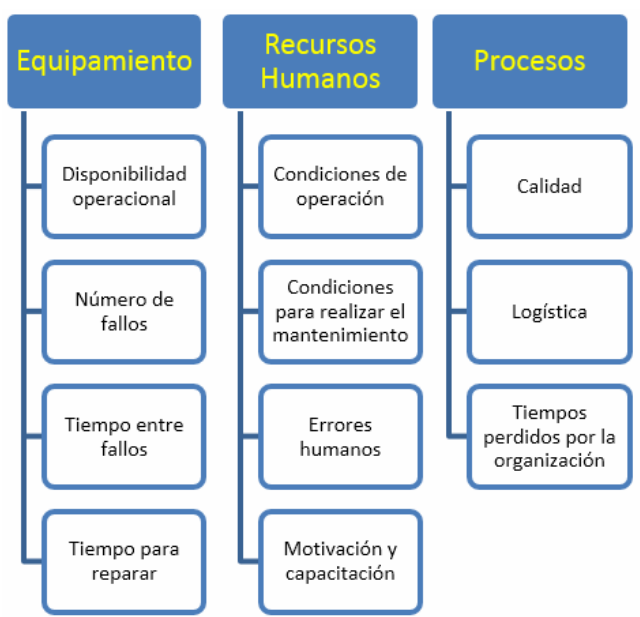

Figura 1. Matriz de confiabilidad operacional.

Tabla 1. Resumen de los componentes del instrumento para validar el concepto de confiabilidad operacional.

\begin{tabular}{|c|l|l|c|}
\hline \multicolumn{1}{|c|}{ Variable de salida } & Dimensiones & \multicolumn{1}{|c|}{ Indicadores } & Ítem \\
\hline \multirow{3}{*}{ Validez del concepto de confiabilidad operacional } & \multirow{2}{*}{ Pertinencia } & Contextualización & $1-2$ \\
\cline { 3 - 4 } & & Actualidad & $3-4$ \\
\cline { 2 - 4 } & \multirow{2}{*}{ Precisión } & Claridad & $5-7$ \\
\cline { 3 - 4 } & & Concordancia & 8 \\
\hline
\end{tabular}


en la toma de decisiones para la mejora de la confiabilidad operacional.

El costo queda como un elemento no tratado de forma directa en la matriz y por ende en el modelo matemático del indicador que se derive de la misma en un futuro. Se observaron diferentes herramientas que abordan dentro de su análisis de confiabilidad operacional el costo, en todas ellas el costo se menciona como elemento más, pero cuando se realiza un análisis exhaustivo de las mismas se observa que este se tiene en cuenta en los análisis costo-beneficio de las acciones de mejora. En esta investigación no se empleó la variable costo, la misma, está concebida su inclusión en la continuación de la investigación, una vez que se disponga de resultados históricos se debe realizar un proceso de optimización, donde una de las variables condicionantes para la implementación de las propuestas de acciones correctivas será la variable costo.

\section{Resultados de la validación del concepto de confiabilidad operacional}

En la Tabla 2 se muestra un resumen de los resultados obtenidos para los restantes elementos que componen el instrumento para la validación del concepto de confiabilidad operacional.
Como se observa en la Tabla 2, todos los indicadores alcanzan valores promedio por encima de 4 . Estos resultados repercuten en que las 2 dimensiones obtengan también valores por encima de 4 y se alcanza un valor total al aplicar el instrumento de 4,26. Todos los resultados obtenidos están por encima de 3,7 por lo que se consideran buenos según lo planteado en la bibliografía $[16,17]$.

Posteriormente se realizó la medición de la confiabilidad del instrumento. Como resultado de esta medición se obtuvo un valor del Alfa de Cronbach de 0,845 , lo que se considera como bueno según los entendidos [15, 18].

La pregunta abierta en la encuesta está formulada como se expone a continuación:

Si lo considera necesario, exponga libremente sus criterios, opiniones, sugerencias y/o posibles modificaciones referentes a la propuesta del concepto de confiabilidad operacional que se le ha presentado.

Para el análisis de la pregunta abierta, las respuestas de los especialistas fueron agrupadas en categorías a partir de su similitud. En la Tabla 3 se muestran los resultados obtenidos.

Tabla 2. Resumen de los resultados de los valores promedios de los elementos del instrumento para validar el concepto de confiabilidad operacional.

\begin{tabular}{|c|c|c|}
\hline Variable de salida & Dimensiones & Indicadores \\
\hline \multirow{4}{*}{$\begin{array}{l}\text { Validez del concepto de confiabilidad operacional } \\
\qquad 4,26\end{array}$} & \multirow{2}{*}{$\begin{array}{l}\text { Pertinencia } \\
\quad 4,15\end{array}$} & $\begin{array}{c}\text { Contextualización } \\
4,22 \\
\end{array}$ \\
\hline & & $\begin{array}{c}\text { Actualidad } \\
4,09 \\
\end{array}$ \\
\hline & \multirow{2}{*}{$\begin{array}{c}\text { Precisión } \\
4,34\end{array}$} & $\begin{array}{c}\text { Claridad } \\
4,37 \\
\end{array}$ \\
\hline & & $\begin{array}{c}\text { Concordancia } \\
4,32 \\
\end{array}$ \\
\hline
\end{tabular}

Tabla 3. Resumen de los resultados de la pregunta abierta.

\begin{tabular}{|l|c|}
\hline \multicolumn{1}{|c|}{ Criterio } & Cantidad \\
\hline $\begin{array}{l}\text { Interés por tener acceso al modelo matemático para la estimación de la } \\
\text { confiabilidad operacional. }\end{array}$ & 18 \\
\hline Preocupación por la necesidad de proponer un nuevo concepto. & 4 \\
\hline $\begin{array}{l}\text { Preocupación por no quedar claramente en el concepto los anteriores } \\
\text { elementos componentes de la confiabilidad operacional. }\end{array}$ & 3 \\
\hline No queda claro el término sinérgico. & 2 \\
\hline
\end{tabular}


De los resultados de la Tabla 3 se observa que las respuestas de los especialistas a la pregunta abierta se agruparon en 4 criterios. En relación con el primero de ellos, 18 especialistas coinciden en estar interesados en tener acceso al modelo matemático que permite el cálculo de la confiabilidad operacional. Esto es debido a no fue explicitado en el instrumento y es uno de los cambios más significativos en el concepto propuesto con respecto a los de otros especialistas [6, 7, 8 y 9]. Para dar respuesta a tal interés, con posterioridad a la publicación del modelo matemático propuesto, al cual da sustento el concepto construido, dicho modelo será dado a conocer a los especialistas consultados.

El resto de las inquietudes planteadas por las especialistas, resumidas en la Tabla 3, no tienen impacto directo en el concepto propuesto. Se consideró que estas tienen una baja incidencia al ser planteados por menos de 5 especialistas. Estos últimos criterios están relacionados con el indicador claridad, en particular la pregunta 5 del cuestionario, la que plantea: ¿en el concepto propuesto está claramente definida la confiabilidad operacional a partir de la integración de sus elementos constituyentes?, esta fue la de más bajo resultado de todas.

Es destacable que en la definición del nuevo concepto se concuerda con los anteriormente expuestos en lo relativo a que un proceso de mejora de la confiabilidad operacional debe sustentarse sobre la base de analizar diferentes elementos tales como:

1. Equipamiento.

2. Procesos.

3. Mantenibilidad.

4. Recursos humanos.

El concepto propuesto solo se basa en tres elementos, equipamiento, recurso humano y el proceso tecnológico. Debe tenerse presente que aquí el elemento equipamiento contiene lo referente a la confiabilidad de los equipos y la mantenibilidad, los que están íntimamente vinculados con la disponibilidad operacional [19].

De los resultados obtenidos, tanto en el valor de la variable de salida como para el valor de confiabilidad, se puede concluir que el concepto de confiabilidad operacional propuesto es aceptado por los especialistas.

\section{CONCLUSIONES}

En esta investigación se definió y validó un nuevo concepto de confiabilidad operacional, que además, da soporte teórico a un indicador que permite su representación numérica.

El nuevo concepto plantea, en forma clara, que la confiabilidad operacional podrá representarse a través de un valor numérico, elemento de máxima importancia debido a que para un correcto proceso de gestión, es de suma importancia contar con indicadores que permitan evaluar el estado de la confiabilidad operacional para así contribuir a la toma de decisiones con una mejor información.

\section{REFERENCIAS}

[1] N. Viego Ariet and J. Romero García. "Preventive tasks for improving aircraft's environmental control system reliability". Revista Cubana de Ingeniería. Vol. $8 \mathrm{~N}^{\circ} 3$, pp. 27-33. 2017. ISSN: 2223-1781.

[2] R.I. Benítez Montalvo, A. Díaz Concepción, et al. "Assessment of components of operational reliability in walk- in freezer". Ingeniería Mecánica. Vol. 19 Nº 2, pp. 78-84. 2016. ISSN: 1815-5944.

[3] G. Kovacevic, J. Dvornik and S. Dvornik. "Analysis of the operational reliability of the rotary cup burner of a marine boiler". International Journal of Mechanical Engineering. Vol. 1, pp. 55-61. 2016. ISSN: 2367-8968.

[4] Ch. Stenström and P. ChristeParida. "Measuring and monitoring operational availability of rail infrastructure". SAGE Journals. Vol. $230 \mathrm{~N}^{\circ}$ 5, pp. 1457-1468. 2016. ISSN: 0954-4097.

[5] D. Zhang and Y. Zhang. "Dynamic decisionmaking for reliability and maintenance analysis of manufacturing systems based on failure effects". Enterprise Information Systems. Vol. $11 \mathrm{~N}^{\circ}$ 8, pp. 1228-1242. 2017. DOI: https:// doi.org/10.1080/17517575.2016.1212406

[6] Pemex Exploración y Perforación. "Aprendizaje virtual. Guía de aprendizaje: Sistema de confiabilidad operacional". 2007. Fecha de consulta: 26 noviembre del 2017. URL: http://www.es.scribd.com/2-SistemaConfiabilidad-Operacional-de-PEP 
[7] C. Hora, S. Dino Calin, et al. "Bb-bc-cg algorithm for operational reliability modeling of hydro generator groups". Procedia Computer Science. Vol. 91, pp. 1088-1095. 2016. ISSN: 1877-0509.

[8] M. Herrera Galán and Y. Duany Alfonzo. "Metodología e implementación de un programa de gestión de mantenimiento". Ingeniería Industrial. Vol. $37 \mathrm{~N}^{\circ}$ 1, pp. 2-13. 2016. ISSN: 1815-5936.

[9] O. García Palencia. "Diagnóstico estratégico de confiabilidad humana del personal de una planta de generación eléctrica”. VII Congreso Mundial de Mantenimiento y Gestión de Activos. León, Guanajuato. 2015.

[10] R. Casares Li, A. Rodríguez Hernández y S. Viña Brito. "Análisis de errores humanos mediante la tecnología TErEH: experiencias en su aplicación". Ingeniería Industrial. Vol. 37, $\mathrm{N}^{\circ}$ 1, pp. 49-58. 2016. ISSN: 1815-5936.

[11] A. Díaz Concepción y L. Villar Ledo. "Análisis bibliográfico de la confiabilidad operacional en sistemas técnicos complejos". Ingeniería Mecánica. Vol. 21 No 2, pp. 77-81. 2018. ISSN: $1815-5944$.

[12] R. Hernández Sampieri. "Metodología de la investigación". 4ta ed. Mc Graw Hill. México DF, México. ISBN: 970-10-3632-2. 2006.

[13] B. Hayes. "Cómo medir la satisfacción del cliente”. Editado E. G. Barcelona, España. ISBN: 84-8088-391-X. 2010.

[14] P. Osadebe. "Construction of Valid and Reliable Test for Assessment of Students".
Journal of Education and Practice. Vol. 6 $\mathrm{N}^{\circ}$ 1. 2015. ISSN: 2222-1735.

[15] J. Bojórquez Molina. "Utilización del alfa de Cronbach para validar la confiabilidad de un instrumento de medición de satisfacción del estudiante en el uso del software Minitab". "Latin American and Caribbean Conference for Engineering and Technology (LACCEI' 2013), Innovation in Engineering, Technology and Education for Competitiveness and Prosperity”. Cancún, México. 2013. Fecha de consulta: 4 de noviembre de 2015. URL: http://www.laccei.org/LACCEI2013-Cancun/

[16] T. Grubessich y R. Stegmaier. "Propuesta metodológica para aumentar el grado de comprensión de un sistema complejo mejorando el proceso de toma de decisión". Dyna. Vol. $92 \mathrm{~N}^{\circ}$ 4, pp. 375. 2017. ISSN: 0012-7353.

[17] S. Domínguez Lara. "Propuesta para el cálculo del Alfa ordinal y Theta de Armor". Revista IIPSI. Vol. 15 N $^{\circ}$ 1, pp. 213-217. 2012. ISSN: 1609-7475.

[18] B. Shane "Utilizing and adapting the Delphi Method for use in qualitative research. International" Journal of Qualitative Methods. Vol. $14 \mathrm{~N}^{\circ}$ 5, pp. 1-6, 2015. DOI: $10.1177 / 1609406915621381$.

[19] A. Dell'Isola and A. Vendittelli. "Operational availability (Ao) of warships: A complex problem from concept to in service phase". MetroAeroSpace. NY, USA. 2015. ISBN: 978-1-4799-7569-3. DOI: 10.1109/ MetroAeroSpace.2015.7180621 